\title{
Study of the chain transfer agent's effect on the butadiene-styrene copolymer's properties based on the Monte-Carlo method
}

\author{
T. Mikhailova ${ }^{1}$, E. Miftakhov ${ }^{2}$, S. Mustafina ${ }^{1}$ \\ ${ }^{1}$ Bashkir State University, 32, Validy Str.,450076, Ufa, Russia \\ ${ }^{2}$ Ufa State Aviation Technical University, 12, K. Marx Str.,450008, Ufa, Russia
}

\begin{abstract}
The microstructural and molecular characteristics of the butadiene-styrene copolymer are investigated depending on the feeding mode of the chain transfer agent in the paper. The study is based on mathematical simulation of the butadiene-styrene copolymerization process by the Monte Carlo method, where the tert-dodecyl mercaptan is used as the chain transfer agent. The dependences of the values of the weightaverage molecular weight, molecular weight distribution and microheterogeneity index on the serial index of the reactor in the cascade are obtained.
\end{abstract}

Keywords: copolymerization; simulation; Monte Carlo method; butadiene; styrene; tert-dodecyl mercaptan

\section{Introduction}

One of the leading branches of modern petrochemistry is the synthetic rubber industry. Butadiene-styrene rubbers obtained by the method of cold emulsion-type polymerization at the temperature of $5-8{ }^{\circ} \mathrm{C}$ are the most common and popular types of synthetic rubbers. These rubbers are widely used in the production of rubber products, but the main area of their usage is the tire industry. That is due to the high technical properties of tires obtained on their basis, as well as to the availability of monomers.

\section{The object of the study}

The production of butadiene-styrene synthetic rubber is carried out in the cascade of connected polymerizers, each of which is a continuous stirred tank reactor. This process is carried out in the continuous mode with a feed of new reagents to the reactor and an extraction of reaction products from it, which provides the continuation of the reactions. But due to the peculiarities of the copolymerization process, the product obtained is heterogeneous in composition and microstructure of the macromolecules.

One of the ways to correct the parameters of the butadiene-styrene copolymer is the effect of the chain transfer agent on the macromolecules growth of the product. The chain transfer agent is continuously added to the reaction mixture at several points of the mechanism of process $[1,2]$. But the optimum quantity of the chain transfer agent's feed and the selection of the feeding mode in the cascade of reactors can only be established by the experiment. In connection with this, it is relevant to study the characteristics of the butadiene-styrene copolymerization product depending on the mode of production and the composition of the reaction mixture.

\section{Methods}

In modern conditions, methods of mathematical modeling are used to study technological processes within the framework of industrial production. The obtained mathematical model allows to predict the physicochemical parameters of the product yielded under exploitation's conditions for a given kinetic scheme and parameters of process.

The kinetic scheme of butadiene-styrene copolymerization was described in paper [3], and an algorithm for simulation the synthesis of butadiene-styrene copolymer in the cascade of reactors by continuous mode has been described in papers [4, 5, 6]. The algorithm of simulation of processes is based on the Monte Carlo method. Since the process under study is continuous, the residence time distribution of the product's macromolecules is taken into account during the simulation.

The choice of this approach was conditioned by the fact that the basis of simulation is an imitation of the formation of copolymer's macromolecules, which allows to store the information about the composition and length of the chains being formed in the dynamics of the synthesis. This, in its turn, allows to determine the values of the product's characteristics at any time during the simulation.

A software was developed to simulate the synthesis of butadiene-styrene copolymer which is carried out in the cascade of stirred tank reactors on continuous mode on the basis of the created model. For the design Visual Studio programming environment was used with $\mathrm{C} \#$ and Visual $\mathrm{C}++$ languages $[7,8]$.

\section{Results and Discussion}

The developed software was used to investigate the effect of the chain transfer agent's feed mode on the characteristics and microstructure of the obtained product. For this purpose, series of computational experiments were performed under the following conditions:

- the load on the cascade by monomers: 3.5 t/h (100 w.p., butadiene -70 w.p., styrene - 30 w.p.), 
- dosage of initiator (pinane hydroperoxide): 0.054 w.p.,

- ratio water / monomers - 220:100,

- working volume of polymerizer $-10.8 \mathrm{~m}^{3}$,

- volumetric flow rate $-9.5982 \mathrm{~m}^{3} / \mathrm{h}$,

- residence time of the reaction mixture in polymerizer $-1.125 \mathrm{~h}$.

In this case, we will use the following chain transfer agent's feed mode: 3 points $\left(1^{\text {st }}\right.$ reactor -0.125 w.p., $3^{\text {rd }}$ reactors -0.027 w.p., $6^{\text {th }}$ reactor -0.027 w.p., $)$ and 2 points of the cascade $\left(1^{\text {st }}\right.$ reactor -0.125 w.p., $6^{\text {th }}$ reactor -0.027 w.p. $)$.

Fig. 1 depicts the dependence of the weight-average molecular weight of the butadiene-styrene copolymer on the index of the reactor in the cascade. The additional feed of the chain transfer agent in the third point of the cascade promotes to slow growth of the values of the product's weight average molecular weight. The molecular weight distribution (MWD) is characterized by an increase in the low molecular weight's fractions and a decrease in the high molecular weight's fractions of the formed copolymer (Fig. 2).

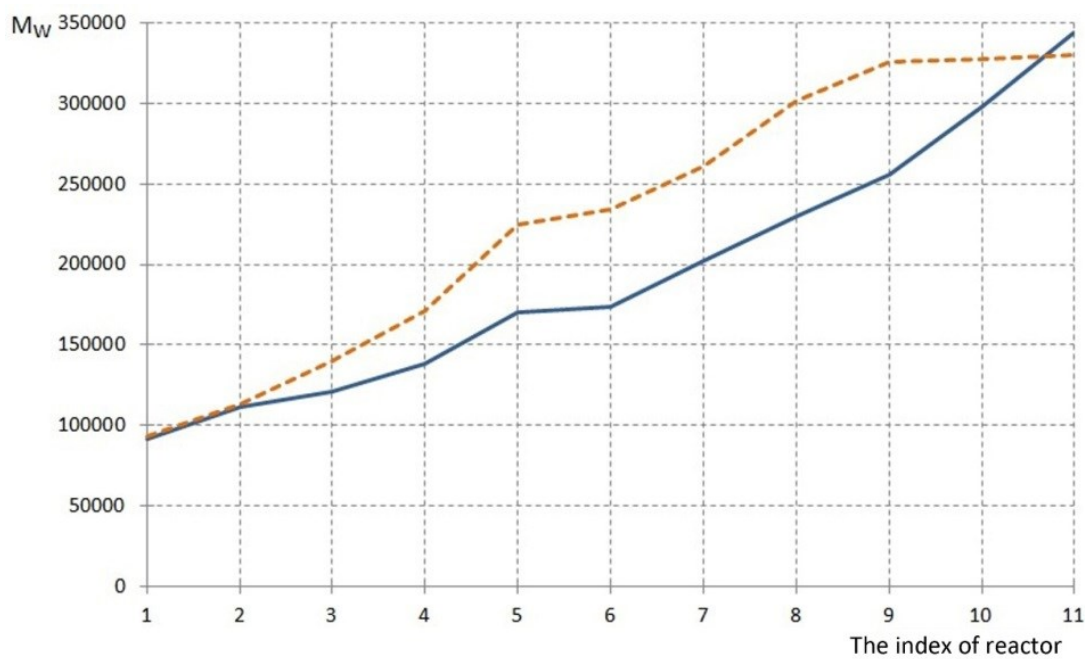

Fig. 1. Changing the weight-average molecular weight of the formed copolymer depending on the index of reactor in the cascade: the dotted line - two-point feed mode, the solid line - three-point feed mode of chain transfer agent.

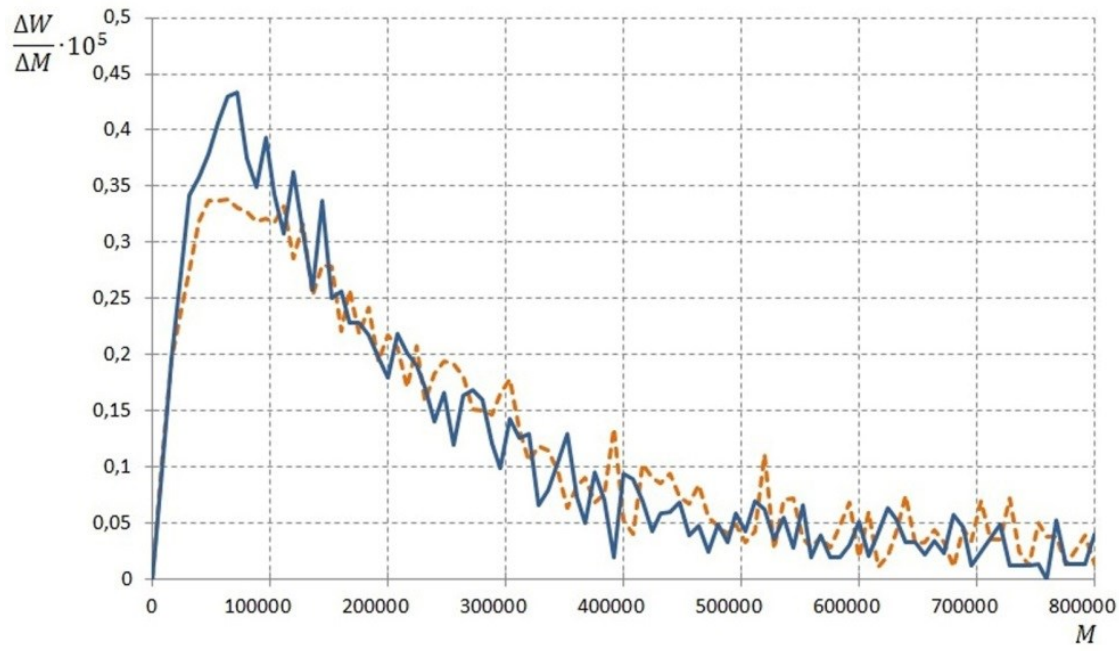

Fig. 2. Differential curve of the molecular weight distribution of the styrene-butadiene copolymer: the dotted line - two-point feed mode, the solid line - threepoint feed mode of chain transfer agent.

The constructed model allows to investigate the sequence of the combination of monomeric units in the formed copolymer's chains. At the same time, the microstructure of macromolecules is usually characterized not by fractions of different sequences of units, but by parameters that represent some of their combinations. This parameter is the microheterogeneity index for the binary copolymer.

If the chain of the binary copolymer can be represented as a sequence of dyads of butadiene-butadiene (BB), butadienestyrene (BS), styrene-butadiene (SB), styrene-styrene (SS), whose fractions are denoted $P_{B B}, P_{B S}, P_{S B}, P_{S S}$, then the microheterogeneity index can be calculated from the following formula:

$$
K_{M}=\frac{P_{B S}}{P_{B} P_{S}},
$$

where the fractions of butadiene and styrene in the chains are calculated according to the formulas:

$$
\begin{aligned}
& P_{B}=P_{B B}+P_{B S}, \\
& P_{S}=P_{S S}+P_{S B} .
\end{aligned}
$$


In Fig. 3 the dotted line shows the dependence of the microheterogeneity index of the copolymer on the index of the reactor in the cascade. Values of the microheterogeneity index vary from 0.98 in the first reactor to 0.87 in the last reactor of the cascade. It characterizes the final product as a statistical copolymer with a tendency to form long blocks. The additional feed of the chain transfer agent to the third point of the cascade helps to narrow the range of variation of the microheterogeneity index and decrease the probability of formation of long blocks: the microheterogeneity index varies from 0.98 in the first reactor to 0.94 in the last reactor of the cascade.

It can be noted that the range of the change in the fraction of butadiene-butadiene homodyads from 0.78 to 0.38 corresponds to the two-point feed mode of the chain transfer agent to versus the range of the change from 0.78 to 0.52 for the three-point feed mode. The fraction of styrene-styrene homodyads varies from 0.02 to 0.2 at the two-point feed mode of the chain transfer agent versus the change from 0.02 to 0.09 at the three-point feed mode. The range of the change in the fraction of butadienestyrene heterodyads at different feed mode of the chain transfer agent varies insignificantly: from 0.2 to 0.42 in the two-point feed mode and from 0.2 to 0.39 in the three-point feed mode. A significant change in the fraction of dyads in the last reactors of the cascade is associated with the total consumption of the chain transfer agent (Fig. 4-5).

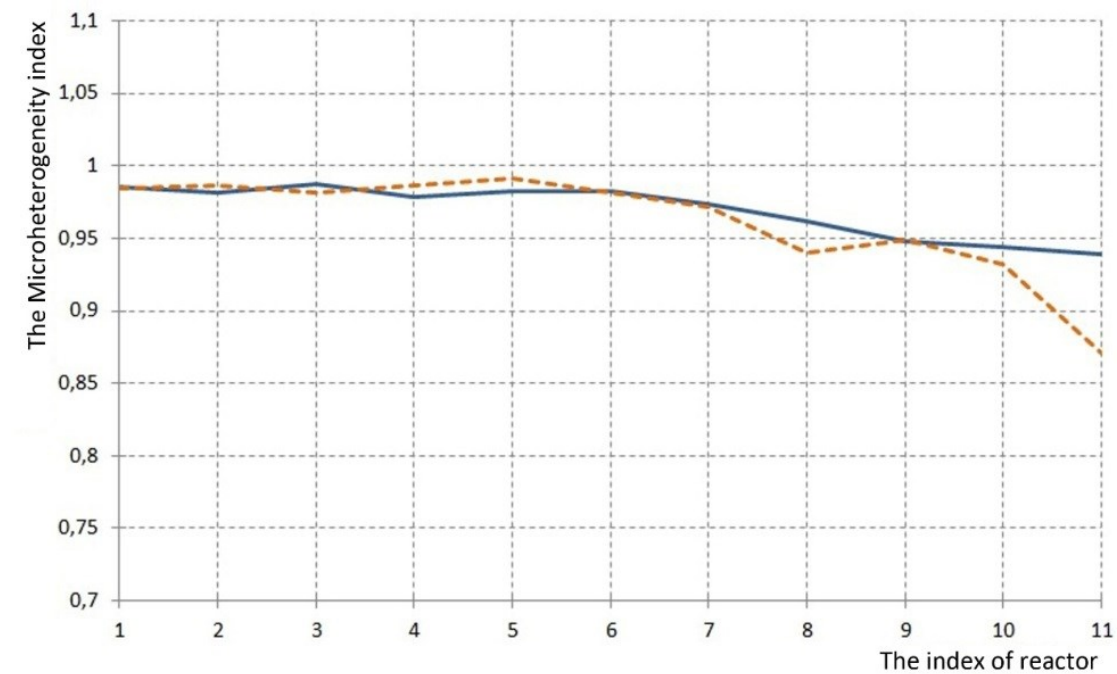

Fig. 3. Changing the microheterogeneity index of the formed copolymer depending on the index of reactor in the cascade: the dotted line - two-point feed mode, the solid line - three-point feed mode of chain transfer agent.

\section{Conclusion}

Simulation of the synthesis of the butadiene-styrene copolymer makes it possible to study the characteristics of the obtained product on the basis of the Monte Carlo method. Since simulation is based on imitating the growth of copolymer's macromolecules and tracking the given processes, it contributes to the accumulation of information on the composition and length of the formed chains in the dynamics of synthesis. This makes it possible to predict and analyze the microstructure of the product. It is established that the fractional feed mode of the chain transfer agent supply leads to the narrowing of the range of the copolymer's microheterogeneity index. The high molecular weight fractions of the copolymer increase during the course of the process, which results in the rigidity of the product obtained on the basis of the copolymer. At the same time, the increase in the content of styrene homodyads in macromolecules contributes to the decrease in the elasticity of the product.

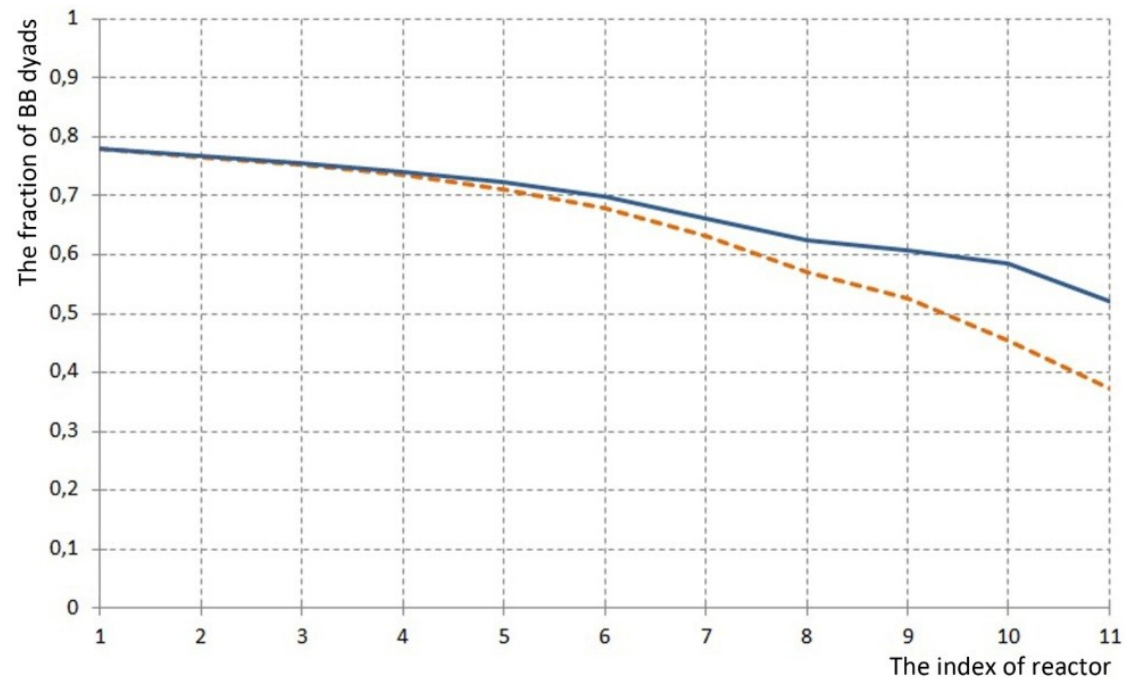

Fig. 4. Changing the values of the butadiene-butadiene homodyads fraction in copolymer chains depending on the index of reactor in the cascade: the dotted line - two-point feed mode, the solid line - three-point feed mode of chain transfer agent. 


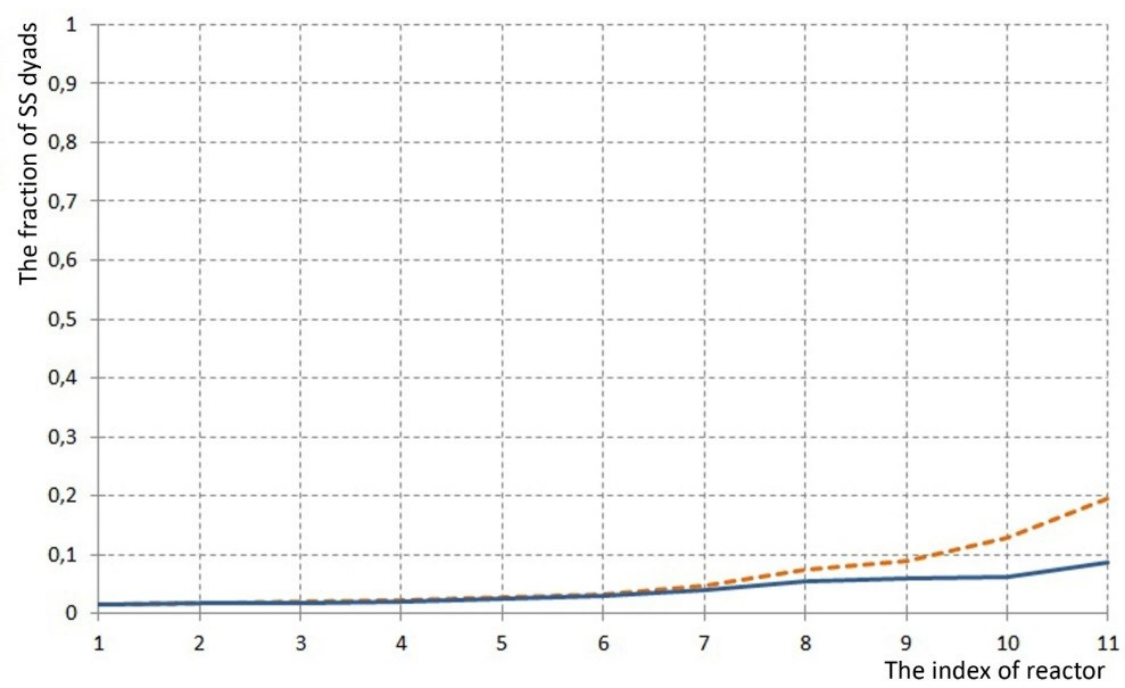

Fig. 5. Changing the values of the styrene-styrene homodyads fraction in copolymer chains depending on the index of reactor in the cascade: the dotted line two-point feed mode, the solid line - three-point feed mode of chain transfer agent.

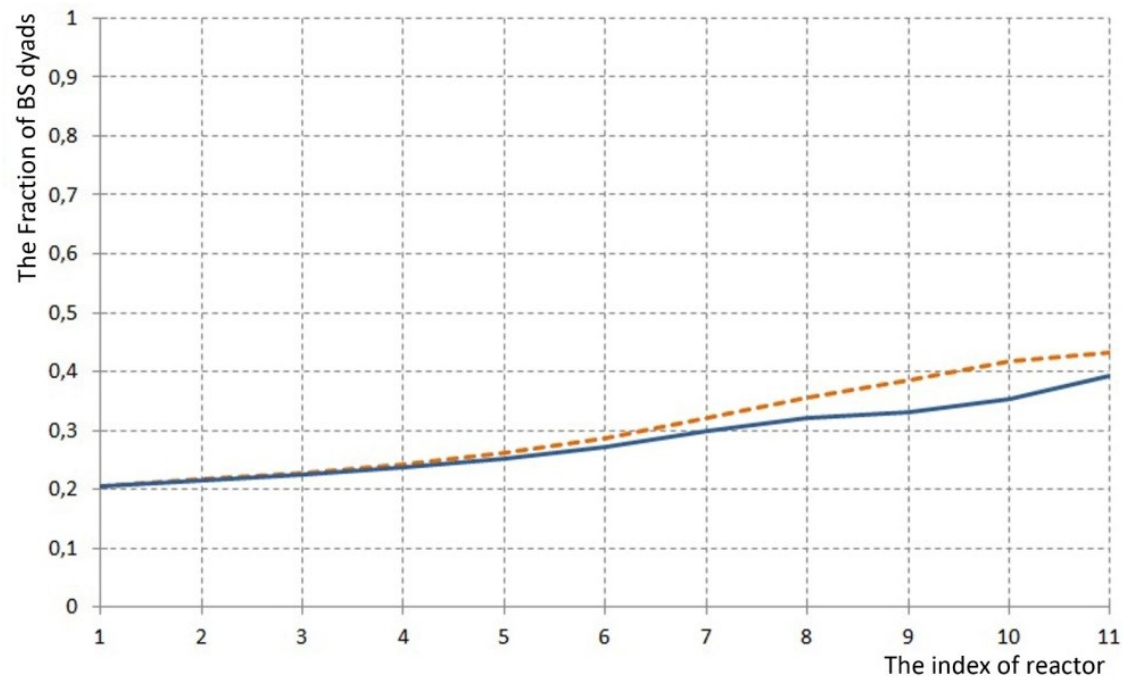

Fig. 6. Changing the values of the butadiene-styrene heterodyads fraction in copolymer chains depending on the index of reactor in the cascade: the dotted line two-point feed mode, the solid line - three-point feed mode of chain transfer agent.

\section{Acknowledgements}

The study was funded by RFBR according to the research projects №16-31-00162 and №17-47-020068 and project No.13.5143.2017/ BCH, carried out by the University in the framework of the State Task of the Ministry of Education of the Russian Federation.

\section{References}

[1] Kirpichnikov PA, Beresnev VV, Popova LM. Album of technological schemes of the main industries of the synthetic rubber. Leningrad: Chemistry, 1986; 224 p. (in Russian)

[2] Averko-Antonovich LA, Averko-Antonovich YuO, Davletbaeva IM, Kirpichnikov PA. Chemistry and technology of synthetic rubber. Moscow: Chemistry, 2008; 357 p. (in Russian)

[3] Mustafina S, Miftakhov E, Mikhailova T. Solving the direct problem of butadiene-styrene copolymerization. International Journal of Chemical Sciences 2014; 12(2): 564-572.

[4] Mustafina S, Mikhailova T, Miftakhov E. Mathematical Study of the butadiene-styrene copolymerization product by the Monte-Carlo method. International Journal of Chemical Sciences 2015; 13(2): 849-856.

[5] Mikhailova T, Miftakhov E, Mustafina S. Mathematical Simulation of the Styrene-Butadiene Rubber's Production in the Cascade of Reactors by the Monte-Carlo Method. International Journal of Chemical Sciences 2016; 14(4): 1865-1876.

[6] Mikhailova T, Mustafina S, Grigoryev I. Numerical study of copolymer composition and compositional heterogeneity during the synthesis of butadiene-styrene rubber. International Journal of ChemTech Research 2017; 10(2): 1031-1036.

[7] Mikhailova T. Computer modeling of styrene-butadiene rubber's production in the cascade of recators by the Monte Carlo method. Control Systems and Information Technologies 2016; 4(66): 64-69.

[8] Mikhailova TA, Miftakhov EN, Mustafina SA. Computer program «CopolMMKforCascade» for simulation of the batch and continuous processes of free-radical butadiene-styrene copolymerization. Federal Service for Intellectual Property (Rospatent). № 2016662302, 07.11.2016. 